\title{
Role of Ultrasound in Assessment of Joint Pain among Hemodialysis Patients
}

\author{
Ahmed Alaa Eldin Ahmed Saad, Ahmad Mohammad Abdulfattah Al-Ashkar, Ahmad \\ Mahmoud Ahmed Mohamed Fahmy, Nabil Fathy Ismael Hassan, Mohammad Sobhy \\ Abdulhameed Abdulkhaliq
}

\author{
Al-Hussein University Hospital, Internal Medicine \& Nephrology Department, Faculty of Medicine, Al-Azhar \\ University, Cairo, Egypt \\ Corresponding author: Mohammad Sobhy Abdulhameed, E-mail: dr_mhsobhey@yahoo.com, Tel: 01008965573
}

\begin{abstract}
Background: Few studies including limited number of patients assessed the rheumatologic effects of hemodialysis (HD) on joints using ultrasonography. Joint ultrasound has been emerged as a cheap noninvasive tool for assessment of joint pain among HD patients. This was the aim of our study to make use of such tool in such life quality threatening complaint. Objective: to determine the role of ultrasound in evaluation of joint pain and its causes among patients on regular HD. Patients and Methods: One hundred and four patients with end stage renal disease (ESRD) who were regular on HD three sessions per week four hours per session were subjected to history taking, complete physical examination stressing on musculoskeletal examination and ultrasonography of painful joints by a an ultrasonography expert. Results: Dialysis related arthropathy (DRA) was not the only cause of joint pain among HD patients but there were diverse causes in different joints. As regard affected joints, knee was the most affected one then came wrist, shoulder, ankle and elbow respectively. As regard causes of joint pain, DRA was the commonest one then came osteoarthritis, nonspecific ultrasonographic findings and few cases showed normal ultrasonographic studies. Conclusion: This study confirmed that joint pain in HD patients has diverse causes not DRA by necessity but other causes must be considered as well as multifactorial etiologies.
\end{abstract}

Key words: Joint ultrasound, Dialysis related arthropathy, Joint pain.

\section{INTRODUCTION}

End-stage renal disease (ESRD) is a significant problem in nearly all countries, and the prevalence has increased dramatically in developing countries and is expected to continue increasing ${ }^{(\mathbf{1})}$. Patients with ESRD may undergo hemodialysis (HD) as a choice of renal replacement therapy (RRT) as a life saving measure and may develop many complications associated with high risk of comorbidities and mortality ${ }^{(2)}$. ESRD patients of all ages have poor quality of life that's why health care professionals caring for HD should target not only patients' life span but improving their quality of life as well ${ }^{(3)}$.

One of the most important qualitative parameters for evaluating patient's quality of life is joint pain ${ }^{(4)}$ which is very common in HD patients ${ }^{(5)}$ and a major cause of depression, disturbed sleep rhythm, impaired dialysis adequacy and noncompliance for $\mathrm{HD}^{\left({ }^{(6)}\right.}$. Causes of pain among $\mathrm{HD}$ patients are diverse and not only of renal origin but even may be due to more than a single cause (5).Our aim was to evaluate joint pain and its causes among $\mathrm{HD}$ patients on regular HD whether it was of renal origin or not by the aid of ultrasound.

\section{PATIENTS AND METHODS}

A total number of 104 ESRD patients who were regular on hemodialysis and having joint pain in Al-Hussein university hospital and nephrology specialized hospital at Galaa military medical complex were subjected to history taking, complete physical examination stressing on musculoskeletal examination and ultrasonography of the painful joint by an expert sonographer. Their ages ranged between $30 \& 70$ years (mean 50). Patients who were 18 years old or younger at time of examination were excluded as well as patients known to have collagen diseases.

All patients underwent musculoskeletal ultrasonography of the painful joints (Knee, wrist, shoulder, ankle and elbow). Ultrasonograms were obtained and interpreted by one researcher (A.F., rheumatologist) using conventional grey-scale ultrasound machine with a 7.5-12 MHz linear transducer. All joints were examined for tendons defects, echogenicity, fluid around and enthesitis, joint effusion and synovial thickness. Knee joint was examined also lemnesci, femoral cartilage sharpness and clarity in addition. Wrist joint was subjected to the same items plus median nerve compression features suggestive of carpal tunnel syndrome (CTS). Shoulder joint was also examined for muscles tears (supraspinatus, subscapularis and infraspinatus) in addition to examination of the acromio-clavicular joint. For ankle joint, it was examined also for tibio-talar joint as well.

As regard laboratory investigations, serum calcium, phosphorus and parathyroid hormones were measured for correlation with joint pain and ultrasonographic features. Serum parathyroid hormone (PTH) was assayed in all patients by the 
Intact Parathyroid Hormone ELISA Kit, normal range: $15-55 \mathrm{pg} / \mathrm{ml}^{(7)}$.

\section{Ethical Issues}

Informed consents were taken from all of the patients included in our study. The study was approved by the Ethics Board of Al-Azhar University.

\section{RESULTS}

We included 104 patients with mean age was 50 and males constituted 53.8\%. Hypertension took the greatest share in causes of end stage renal disease (ESRD). Next to it came diabetes mellitus (DM) after them came the remaining causes as chronic pyelonephritis, non-steroidal anti-inflammatory drugs (NSAIDs) abuse, unknown causes and obstructive uropathy respectively. Hemodialysis duration ranged from 2 to 15 years (mean 7).

Patients who were regular on hemodialysis at Al-Hussein university hospital dialysis units and Galaa military medical complex were 213 patients. 104 patients (48.82\%) had joint pain (shoulder, elbow, wrist, knee or ankle). They were subjected to musculoskeletal examination of the painful joint. Among all 104 patients, 36 patients (34.61\%) had knee pain, 24 patients $(23.07 \%)$ had wrist pain, 20 patients $(19.23 \%)$ complained of shoulder pain, 12 patients $(11.53 \%)$ had ankle pain and only 8 patients (7.69\%) had elbow pain (Figure 1).

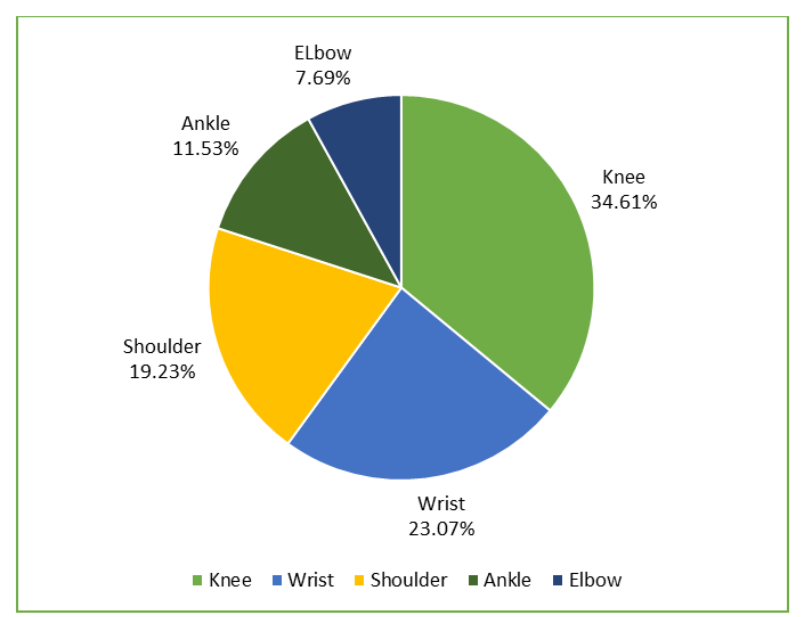

Figure (1): Distribution of joint pain among patients.

Restriction of movement was present in 28 patients (26.92\% of patients) with $12(42.85 \%)$ of which in wrist, 8 patients $(42.85 \%)$ in shoulder, 4 patients $(42.85 \%)$ in knee and ankle separately. Tenderness was present in 80 patients (76.92\%), 32 of which $(40 \%)$ were at knee, 20 patients $(25 \%)$ at wrist, 16 patients $(20 \%)$ at shoulder, 8 patients $(10 \%)$ at ankle and 4 patients (5\%) at elbow.

Regarding laboratory investigations, same number of included patients were taken as controls (104 HD patients without joint pain) for correlation with serum calcium, phosphorus and PTH (Table 1).

Table (1): Laboratory findings in both groups.

\begin{tabular}{|l|l|c|c|}
\hline \multicolumn{2}{|c|}{} & Subjects & Controls \\
\hline \multirow{3}{*}{ PTH } & High & 47 & 27 \\
\cline { 2 - 4 } & Normal & 33 & 62 \\
\cline { 2 - 4 } & Low & 8 & 23 \\
\hline \multirow{3}{*}{ PO4 } & High & 52 & 73 \\
\cline { 2 - 4 } & Normal & 27 & 29 \\
\cline { 2 - 4 } & Low & 5 & 14 \\
\hline \multirow{3}{*}{ Ca } & High & 10 & 21 \\
\cline { 2 - 4 } & Normal & 65 & 82 \\
\cline { 2 - 4 } & low & 9 & 13 \\
\hline
\end{tabular}

Among the 36 patients with knee pain, $66.66 \%$ (24 subjects) were attributed to osteoarthritis as evidenced by presence of osteophytes, 25\% (9 subjects) due to dialysis related arthropathy as denoted by CPPD. The remaining $8.3 \%$ (3 subjects) were not diagnosed as their findings were nonspecific.

Regarding wrist joint, CTS was present in $87.5 \%$ (21 subjects) with CPPD positive in $85.7 \%$ of them which is matching with DRA. The remaining cases showed nonspecific findings.

For shoulder joint, $80 \%$ of patients with shoulder pain (16 subjects) had ultrasonographic features suggestive of amyloidosis with minimum and maximum hemodialysis duration of 5 and 15 years respectively. Impigmentation test was positive in $81.25 \%$ (13 subjects). Apprehension and Scarf's test were positive in $56.25 \%$ ( 9 subjects). The remaining 20\% (4 subjects) had partial thickness tear in supraspinatus which is not related to hemodialysis. They are all manual workers.

As regard ankle joint, $83.33 \%$ (10 subjects) of patients had material deposition and joint effusion. Doppler signal and synovial thickening were present in 58.33\% (7 subjects). $41.66 \%$ (5 subjects) had Achilles tendon bursae. This means that $83.33 \%$ (10 subjects) of ankle pain was attributed to DRA while the remaining cases had nonspecific radiological findings.

For elbow joint, $50 \%$ of patients had non DRA inflammatory characteristic findings in the form of hypoechogenicity of both common extensor origin and triceps tendon, enthesitis and fluid around triceps 
tendon. The remaining subjects' ultrasonography showed unremarkable findings.

\section{DISCUSSION}

Dialysis-related amyloidosis (DRA) is a disorder caused by tissue deposition of beta2microglobulin (b2M) as amyloid fibrils ${ }^{(8)}$. The tissue deposition of amyloid detected histologically occurs much earlier than any clinical or radiographic manifestations of the illness. A prospective postmortem study found joint amyloid deposition in $21 \%$ in patients receiving hemodialysis for less than 2 years, $50 \%$ at $4-7$ years, $90 \%$ at $7-13$ years, and $100 \%$ at more than 13 years ${ }^{(9)}$. The pathogenesis and pathophysiology of DRA are probably multifactorial and have been associated with the duration of renal failure, patient's current age, patient's age at initiation of hemodialysis, duration of hemodialysis, bioincompatibility of dialysis membranes ${ }^{(\mathbf{1 0})}$, less residual renal function, and the coexistence of high-turnover renal osteodystrophy ${ }^{(11)}$. Dialysisrelated amyloidosis is characterized by painful stiff joints, usually first involving the shoulder, and less commonly the hands, wrists, knees, and other large joints. Involvement tends to be bilateral and symmetrical, and is frequently associated with the carpal tunnel syndrome (CTS) and tenosynovitis ${ }^{(12)}$.As clinical symptoms of DRA are nonspecific, they may easily be misinterpreted as other joint diseases ${ }^{(13)}$. Typical specific radiological amyloid bone cysts are a late event. Different studies observed that capsulosynovial swelling precedes the development of characteristic bone cysts ${ }^{(14,}$ ${ }^{15}$. They are hypothesizing that amyloid infiltration of synovial membrane and tendons ${ }^{(\mathbf{1 6}-18)}$ might be accessible to ultrasonographic assessment (19). Ultrasonography (US) has been suggested as the modality of choice in evaluating DRA of the shoulder. Thickening of the supraspinatus tendon and thickening of the long head of the biceps tendon, both in the appropriate clinical setting, have correlated excellently with DRA of the shoulder ${ }^{(15,20,21)}$. Biopsy is nearly always required for definitive diagnosis. However, because histologic confirmation is not always possible and increased serum b2M level is not diagnostic, imaging findings combined with history and clinical findings are usually used for the assessment of musculoskeletal involvement by DRA ${ }^{(22)}$.
To our knowledge, only one study has been performed about this topic entitled Sonographic features suggestive of amyloidosis in hemodialysis patients: relation to serum beta2-microglobulin at Minia University, Egypt 2014. This study was limited to shoulder arthropathy in HD patients. This study included 32 patients with shoulder pain. Male to female ratio was 9:7. In our study, the ratio was 6:5. In our study, restriction of shoulder movement was present in 8 patients out of 20 shoulder pain patients $(40 \%)$ while 5 of $32(15.6 \%)$ in the other study.

In the present study, among the 104 examined subjects, $56(53.84 \%)$ only showed clinical and radiological findings suggestive of DRA while the remaining $(46.15 \%) 48$ subjects' joint pain was attributed to other causes rather than hemodialysis. The most DRA affected joint was wrist showing 21 cases (37.5\%), shoulder with 16 subjects $(28.57 \%)$, ankle with 10 subjects $(17.85 \%)$ and knee with 9 subjects (16.07\%) of subjects with DRA. Elbow ultrasonography was unremarkable regarding DRA.

\section{CONCLUSION}

Our study demonstrated that among the 104 examined subjects, $56(53.84 \%)$ only showed clinical and radiological findings suggestive of DRA while the remaining (46.15\%) 48 subjects' joint pain was attributed to other causes rather than hemodialysis (osteoarthritis, tendon tears, other causes).

The most DRA affected joint was wrist showing 21 cases $(37.5 \%)$, shoulder with 16 subjects (28.57\%), ankle with 10 subjects $(17.85 \%)$ and knee with 9 subjects $(16.07 \%)$ of subjects with DRA. Elbow ultrasonography was unremarkable regarding DRA (Figure 2).

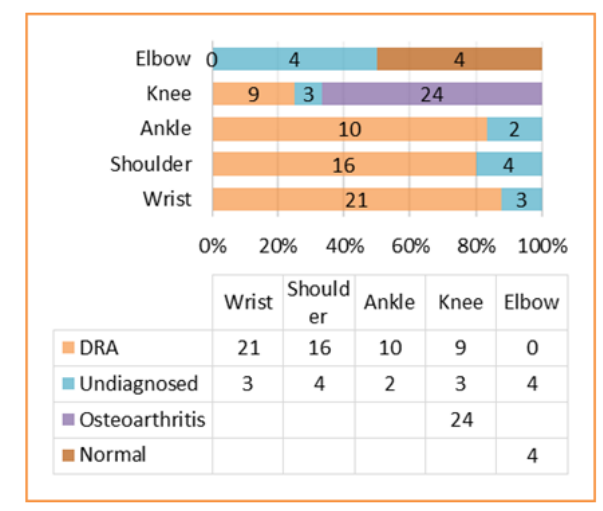

Figure (2): Distribution of joint pain causes. 


\section{REFERENCES}

1. Hoerger TJ, Simpson SA, Yarnoff BO et al. (2015): The future burden of CKD in the United States: a simulation model for the CDC CKD Initiative. Am J Kidney Dis., 65(3):403411.

2. Kovesdy CP, Mehrotra R, Kalantar ZK et al. (2008): Chronic kidney disorders mineral and bone disease - calcium obsession, vitamin $\mathrm{D}$, and binder confusion. Clin $\mathrm{J}$ Am Soc Nephrol., 3(1):168-173.

3. Hsu HJ, Yen CH, Hsu KH et al. (2014): Factors associated with chronic musculoskeletal pain in patients with chronic kidney disease. BMC Nephrol., 15:6.

4. Lapane KL, Quilliam BJ, Benson C et al. (2015): Impact of noncancer pain on healthrelated quality of life. Pain Pract., 15(4):333-342.

5. Davison SN (2003): Pain in hemodialysis patients; prevalence, cause, severity, and management. Am J Kidney Dis., 42(6):12391247.

6. Santoro D, Satta $E$ and Messina $S$ (2013): Pain in end-stage renal disease: a frequent and neglected clinical problem. Clin Nephrol., 79(1):S2-S11.

7. Kruger $\mathrm{L}$, Rosenblum $\mathrm{S}$, Zaazra $\mathrm{J}$ et al. (1995): Intact PTH is stable in unfrozen EDTA plasma for 48 hours prior to laboratory Analysis. Clin. Chem., 41:6.

8. Dember LM and Jaber BL (2006): Dialysisrelated amyloidosis: late finding or hidden epidemic? Semin Dial., 19(2):105-9.

9. Jadoul M, Garbar C, Noe“ I H et al. (1997): Histological prevalence of beta 2microglobulin amyloidosis in hemodialysis: a prospective post-mortem study. Kidney Int., 51(6):1928-32.

10. Danesh F and Ho LT (2001): Dialysis-related amyloidosis: history and clinical manifestations. Semin Dial., 14:80-5.

11. Schulman G and Burkholder JF (2001): Dialysis related disorders: editor. Hospital physician (rheumatology board review manual), 5(2): 2-10.

12. Bardin $T$ and Kuntz D (1987): The arthropathy of chronic haemodialysis. Clin Exp Rheumatol., 5:379-86.
13. Al-Taee IK, Al-Safar JJ, Al-Falahi YS et al. (2003): The clinical significance of b2microglobulin in end-stage renal disease. Saudi J Kidney Dis Transpl.,14(4):492-6.

14. Jadoul M, Malghem J, Vande Berg B et al. (1993): Ultrasonographic detection of thickened joint capsules and tendons as marker of dialysis-related amyloidosis: a crosssectional and longitudinal study. Nephrol Dial Transplant, 8(10):1104-9.

15. Van YC, Honon B, Vandenbroucke JM et al. (1988): Dialysis amyloidosis. Advances in nephrology. Year Book Medical Publishers, Chicago.

16. Mack LA, Nyberg DA and Matsen FA (1988): Sonographic evaluation of the rotator cuff. Radiol Clin North Am., 26(1):161-77.

17. Morita T, Suzuki M, Kamimura A et al. (1985): Amyloidosis as a possible new type in patients receiving long-term haemodialysis. Arch Pathol Lab Med., 109:1029-32.

18. Lusenti T, Borgatti PP, Lindner $G$ et al. (1991): Multiple tendineal tumoral amyloid masses in a patient on longterm dialysis. Nephron, 57:243-4.

19. Vandenbroucke JM, Jadoul M, Maldague B et al. (1986): Possible role of dialysis membrane characteristics in amyloid arthropathy. Lancet, 1:1210-1.

20. McMahon LP, Radford J and Dawborn JK (1991): Shoulder ultrasound in dialysis related amyloidosis. Clin Nephrol., 35:227-32.

21. Cardinal E, Buckwalter KA, Braunstein EM et al. (1996): Amyloidosis of the shoulder in patients on chronic hemodialysis: sonographic findings. AJ Am J Roentgenol., 166(1):153-6.

22. Kiss E, Keusch G, Zanetti M et al. (2005): Dialysis-related amyloidosis revisited. AJR Am J Roentgenol., 185(6):1460-7. 